\title{
Chinese Technical Communicators' Opinions on Cultural Differences between Chinese and Western User Manuals
}

\author{
Qian Li \\ University of Twente \\ q.li@utwente.nl
}

\author{
Joyce Karreman \\ University of Twente \\ j.karreman@utwente.nl
}

\author{
Menno de Jong \\ University of Twente \\ m.d.t.dejong@utwente.nl
}

\begin{abstract}
One of the most significant challenges that technical communicators face in today's globalizing world is how to meet the requirements of target groups from different cultures. Translation and localization of user documentation are important activities in the field of technical communication. Previous studies have shown that there are differences between content, structure and style of user manuals from different cultures. Especially the differences between Chinese and Western manuals seem to be significant. It is not known, however, if and how technical communicators take cultural differences into account when designing manuals. To explore the opinions of Chinese technical communicators, twenty of them were interviewed. The results show that the majority of the interviewees assumed that Chinese and Western manuals differ from each other in many aspects (content, structure, style, visuals) and that Chinese and Western users have a different approach to manuals. Overall, the interviewees considered it important to target manuals to the culture of the users, but they experience several obstacles to do this, such as the obligation to use Western style sheets, the costs related to localizing and a lack of knowledge. Although the results may not be generalizable, because most interviewees worked in the software and telecommunication industry, they clearly show that there is a lack of knowledge about how to localize user manuals.
\end{abstract}

Index Terms - Chinese technical communicators, cultural differences, interview study, user manuals.

\section{INTRODUCTION}

Many technical communicators work in international environments nowadays. A significant challenge facing them is to communicate effectively with colleagues from various cultural backgrounds and to design user documents that are in line with the requirements of customers from different cultures. A lack of understanding of the cultural differences may result in miscommunication. In this article, we focus on differences between Chinese and Western user documentation. Many articles have been published about differences between communication in China and in the Western world; sometimes comparing these two cultures, sometimes comparing specific groups within these cultures. Based on the results of these studies, it is concluded that literally translating functional documents, such as user instructions, from one language into another does not result in documents that are optimally usable [1, 2, 3]. These documents need to be localized, adjusted to the target group's culture.

Literature in the field of technical communication shows that target groups from China and the Western world may have different perceptions of 'good' or 'effective' documents [4, p. 386]. Their preferences seem to differ with regard to, among other things, the structure of a document [2] and the comprehension of the visuals [5]. The opinions of Chinese and Western target groups seem to differ on the acceptability and usability of documents as well [6], and even on their attitudes towards what is ethical or legal [4]. In addition, studies also reveal different design strategies between Chinese and Western manuals [1, 7].

However, little is known about technical communicators' perspective on cultural differences between user documentation and on how to face the challenge of localizing these documents. This article aims to shed more light on the opinions of Chinese practitioners in the field of technical communication. Their ideas about the differences between Chinese and Western target groups of user documentation, about the differences between user documentation designed in different cultures and about their localization activities are investigated.

\section{LITERATURE REVIEW: DIFFERENCES BETWEEN CHINESE AND WESTERN USER MANUALS}

Previous studies in the field of technical communication on differences between China and the Western world have primarily focused on differences between the content, 
structure and style of user instructions, and the use of visuals in the instructions.

With regard to the content of user instructions, study results have indicated that Chinese manuals include more information types that are not directly related to tasks or actions the user needs to perform. For example, Chinese documents seem to include more advertisements and welcoming messages [8]. Furthermore, it seems not unusual that Chinese manuals include information for different target groups. They are not always only targeted to end users, but sometimes also to technicians, since another study showed that more technical information is to be found in Chinese manuals [9]. A recent content analysis study in which 50 Chinese user manuals for a variety of household appliances were compared with 50 user manuals from Western origin confirmed these earlier results [10].

Study results with regard to the structure of Chinese and Western documents are not conclusive. Some researchers concluded that in Chinese documents less structure markers such as headings and lists are used, because Chinese readers try to connect everything together [1]. However, other study results indicated that similar organizational strategies are applied in Chinese and Western documents [8]. The results of the recently conducted content analysis study showed a more nuanced view of differences regarding structure: About the same number of structural elements is used in user instructions from both cultures, but these elements are less explicitly and systematically used in Chinese user instructions. The authors concluded that, overall, Chinese manuals have a 'fuzzier' structure than Western manuals [10].

The most significant difference related to the style of user instructions that is proposed by earlier studies is that the style of Chinese manuals is more implicit and less direct than the style of Western manuals. This is exemplified by shorter task descriptions [9], less clearly identified actions [11], indirect suggestions instead of direct recommendations [1], and less specified tools for tasks [9]. Another difference mentioned in earlier studies is related to politeness. It is suggested that the preferred style of Chinese documents is formal, and that formality is used to express politeness and respect. The preferred style of Western documents is suggested to be relatively informal; a conversational tone is expected to be more appropriate for Western documents [1].

The results of several studies have indicated that more visuals can be found in Chinese user instructions than in Western instructions [5, 7]. Furthermore, study results indicated that the relation between visuals and the accompanying text is less clear in Chinese user instructions $[1,9]$. However, the results of the previously mentioned content analysis could not confirm these earlier results: no differences were found between the number of visuals and the extent to which visuals are integrated with the text. The results of this recent study, however, showed that different types of visuals are used in Chinese user instructions than in Western user instructions [10, 12]. More cartoon-like figures are used in Chinese manuals and more detailed pictures of how a task should be performed are found in Western manuals. These results indicate that Chinese manuals also have an entertaining function, while Western manuals' functions are restricted to informing and instructing.

Summarizing, studies have indicated that there are many differences between Chinese and Western manuals, but the study results are not always conclusive. Apart from this, it is unclear if Chinese technical communicators recognize these differences, what their opinions are about these differences and how they take these differences into account when designing manuals for different target groups from different cultures.

\section{METHOD}

\section{Participants}

Twenty Chinese practitioners in the field of technical communication voluntarily participated in this study; they did not receive an incentive. They were interviewed by the first author of this paper, who is a native speaker of Chinese. The interviewees were recruited via social media: WeChat groups related to the field. They were selected based on two criteria. The first was that they should have experience writing and/or translating manuals in Chinese as well as in English, to ensure that they were familiar with intercultural target groups. The second criterion was that they should have been working in the field of technical communication for at least two years to ensure they that had sufficient experience and knowledge.

Seven male and 13 female practitioners participated. They were on average 34 years old $(S D=5.5)$. They had been working in the field for 6.5 years on average (SD = 2.8). The primary task of 17 out of the 20 interviewees was to write manuals, the other three interviewees' main task was to translate manuals. Eight interviewees acted as a team leader or manager as well. Almost half of the interviewees (9) had an educational background in engineering, about the same number (8) had an interdisciplinary educational background, and only three interviewees had a language oriented educational background. The majority (15) of the interviewees held a master's degree, the others held a bachelor's degree.

\section{Procedure and interview themes}

All interviews were conducted face-to-face in mainland China. Before the interview started, the interviewees were informed about the interview topics and goals, there were told that the interview would be audio recorded, and they were asked to sign an informed-consent form.

The interview was conducted in a semi-structured way. In all interviews, questions about four different themes were asked, but not necessarily in exactly the same order. The first, broad theme encompassed several questions to 
get insight in the interviewees' opinions on what they considered to be their most important professional skills and in their opinions on the relation between technical writing, localizing and translating. The second theme encompassed questions about the assumed differences between Chinese and Western manuals, including, but not limited to differences related to the content, structure and style of manuals, and the use of visuals. The third theme encompassed questions about the assumed differences between Chinese and Western manual users. The final theme was about translation and localization. Questions were asked about issues that interviewees considered to be important when being involved in translation and localization activities.

At the end of the interview, the interviewees were asked to write down some demographic information (age, work experience, educational background). The interviews took one hour and a quarter (75 minutes) on average, ranging from 52 minutes to 125 minutes.

\section{Data analysis}

All interviews were transcribed verbatim by the interviewer (first author). Then, the data were analysed using an inductive approach. The first author started with reading through a number of interviews, tagging fragments that seemed to be relevant. Gradually, she generated a codebook consisting of codes related to the interview themes and some other emerging topics. The codebook was finalised in consultation with the other authors. The codebook comprised five main categories:

- $\quad$ Differences between Chinese and Western users;

- Differences between Chinese and Western manuals;

- Relations between technical writing and localization;

- Important issues in localization;

- Work practice experiences related to localization.

Apart from the five main categories, a category 'remaining topics' was defined. All categories comprised several subcategories. An example of a subcategory within the main category of 'differences between Chinese and Western users' is 'the extent to which users rely on manuals.' The main category of 'differences between Chinese and Western manuals' comprised the subcategories 'content', 'structure', 'style' and 'visuals.' Examples of subcategories of the category 'relations between technical writing and localization' are 'the two field are integrated' and 'the two fields are separated'. Examples of subcategories of the two last main categories are respectively 'translation of concepts' and 'use of tools and style sheets.'

After finalising the codebook, two complete interviews (67 fragments) were randomly selected to be coded, using the finalised codebook, by the first author and a second, independent, coder, who was also a native speaker of Chinese. Since the results showed that the interrater reliability was good (Cohen's Kappa $=0.84$ ), the codebook was not changed and the other interviews were coded by the first author, using the software application ATLAS.ti.

\section{RESULTS}

\section{Technical communicators' opinions on the importance of cultural skills}

Out of the twenty interviewees, 19 were of the opinion that technical writing and localization are closely related. Some of them considered the two fields to be merged and did not see any clear borders between them. More than half of the interviewees stated that technical writers should take localization issues into account when writing a manual. They thought that for writing a manual and for localizing, the same skills were needed: the ability to use language and product knowledge to help the targeted users understand the product. They considered cultural skills to be very important nowadays, to be able to optimally target a manual to the intended users:

The skill of cross-cultural understanding is quite essential. Only by considering the habits of the local society is it possible to design or localize manuals that are more acceptable to the users. (\#06, Male, 10 years of experience)

Localization [...] is not simple language conversion but transfer of a cultural context. (\#N15, Male, 6 years of experience)

Five interviewees mentioned that improper localization could directly contribute to a company's failure to enter a foreign target market:

Some Chinese companies had trouble in entering the overseas market because foreign users could not understand the manual. (\#09, Female, 6 years of experience)

Most interviewees considered cultural skills important, not only for being able to write manuals for users from different cultures, but also to be able to function as a competent professional. For them, it was likely to participate in virtual teams and collaborate with professionals from other cultures. About half of the interviewees had experienced differences in communication practices across cultures:

Chinese R\&D professionals expect technical writers to extract meanings from the conversation with them, while Western R\&D professionals describe what they need into detail. So, methods need to be changed when 
talking to people from different cultures. (\#19, Female, 10 years of experience)

So, the interviewees were aware of cultural differences between China and Western cultures and they considered it important to take these differences into account when designing manuals and when collaborating with colleagues from other cultures.

\section{Technical communicators' opinions on differerences between Chinese and Western manual users}

Most interviewees shared the opinion that Chinese people had different expectations from manuals than Western people. Overall, they assumed that Western users prefer long and detailed manuals and that Chinese users preferred shorter manuals. Fourteen interviewees stated that they considered Chinese users to be less dependent on manuals for operating devices than Western users. They thought that in general, Western users read manuals carefully and that Chinese users do not read them at all. They also assumed that when Western users encountered a problem while operating a device, they would consult the manual to try to solve their problem and that Chinese users would try to find someone who could help them or call a help desk. They related this to the difference between the individualistic Western cultures and the collectivistic Chinese culture, where relationships are more important:

The reason why Chinese people prefer to call the help desk is that they can incidentally promote friendly relations with the service personnel. (\#09, Female, 6 years of experience)

Related to the previous assumption is the assumption that Western users prefer comprehensive manuals that are well-structured, so they can easily find the information they need. Chinese users, on the other hand, are supposed to prefer shorter manuals that they can read entirely. Three interviewees made remarks similar to this one:

Western users are accustomed to navigating contents by the index while Chinese users generally read the manual by sequence. (\#01, Male, 10 years of experience)

Another related assumption is that six interviewees thought that for Western users, manuals were purely functional documents that they used to be able to operate a device properly, while Chinese users expected to be entertained by a manual:

It is enjoyable to see beautiful visuals in a manual. But I think Western users only read manuals to solve problems and that they would never regard manuals to be entertaining. (\#03, Female, 3 years of experience)
Five of the interviewees referred to another difference related to the expectations of manual users. They explained that in China, it is the users' responsibility to understand the manual, while in the Western world, technical communicators bear the responsibility to design manuals that are easy to use. They considered this to be the main reason for Western manuals to be long and comprehensive, and Chinese manuals to be short and less detailed.

\section{Technical communicators' opinions on differences on Chinese and Western manuals}

The interviewees spent most time describing perceived differences between Chinese and Western manuals. They mentioned several differences related to the content of manuals, to the structure, to the writing style and to the use of visuals.

Content Overall, the interviewees assumed that Western manuals were more comprehensive and detailed than Chinese manuals, but they did not mention many specific differences with regard to the content of manuals. About half of the participants stated that due to different laws and regulations, legal and safety information in Chinese manuals differed from legal and safety information in Western manuals. Furthermore, somewhat contradictory to the overall assumption that Western manuals are more comprehensive, two interviewees stated that there are more technical terms and technical details present in Chinese manuals. Their explanation was that Chinese manuals are often written by technicians. Another interviewee pointed out that she thought that Chinese manuals included more information that is not directly relevant to operating the device. This is in line with the assumption that Chinese users would like to be entertained reading a manual.

Structure The interviewees' opinions on differences between the structure of Chinese and Western manuals were more articulated. Thirteen interviewees stated that Chinese and Western manuals were differently structured. Six of them stated that the structure of Chinese manuals was less explicitly marked and therefore, less logical, because less conjunctions and other structure markers are used in Chinese:

In English, several conjunctions such as 'while' and 'then' are used to indicate the sequence of events, but in Chinese, steps are described without these words. Therefore, the manual might be less logical to the user. (\#12, Female, 11 years of experience)

Two others agreed with them about the assumption that less structure markers were used, but they did not think that this resulted in a less logical structure. They thought that both ways of structuring the manuals were consistent with their own culture. 
Four other interviewees mentioned that the overall structure of Chinese documents is different from the structure of Western documents:

The structure is totally different. Western manuals show the main idea at first, but Chinese show it last. (\#09, Female, 6 years of experience)

These interviewees assumed that Chinese user documentation is inductively structured (first argumentation/description, followed by the main idea/conclusion) and Western user documentation deductively (first the main idea/conclusion, followed by argumentation/description). However, five others did not agree. They were of the opinion that all users prefer the same structure: first familiar information, then unfamiliar information. This helps readers to build up a mental model of the text:

Manuals should be organized from easy to difficult [...]. Then, it is easier for the reader to accept and understand a new concept. (\# N08, Female, 2 years of experience)

Five interviewees characterized the differences with regard to structure in a different way. They assumed that Chinese manuals were more product-oriented and that Western manuals were more task-oriented. This assumption is related to the assumption that Western users primarily use manuals to solve problems and that they need to be able to find the information they need quickly, while Chinese users expect that they must read a complete manual to be able to understand how to operate a device:

The way of thinking of Western users is problembased and they expect to be able to solve problems with the help of the manual's explicit explanations. We, Chinese people, think more product-based and we expect to understand what our task is, based on the description. (\#22, Male, 6 years of experience)

Style Not many differences with regard to style were mentioned. Some participants described the Chinese writing style as somewhat vaguer and less precise than the Western style. This assumption is strongly related to assumptions on structural differences, as the use of less conjunctions in Chinese or the presence of more detailed task descriptions in Western manuals.

Two interviewees referred to another style difference. They perceived Chinese manuals as more polite:

In Chinese manuals, 'please' is used many times for modesty and politeness, but writers of Western manuals show equal status with the user. (\#03, Female, 3 years of experience)
Visuals With regard to visuals, the majority of the interviewees assumed that all users, Chinese and Western, preferred visuals in manuals over text, because they thought that visuals would reduce complexity:

All people prefer to read a simple manual, so the ideal manual is one with less text and more graphics. (\#06, Male, TW, 10 years of experience)

Four interviewees assumed that there are different types of visuals in Chinese manuals than in Western manuals. More specifically, they thought that there are more cartoon-like visuals in Asian manuals. They indicated that the use of cartoons originates in Japanese documents, but that they are also commonly used in other eastern Asian cultures. In addition to this, several remarks were made about the use of different colours in Chinese and Western user documentation, because of the different meanings of colours in various cultures.

Furthermore, five interviewees stated that visuals in Chinese manuals are often less well-designed and professional, because there are less graphic design tools available in China for technical communicators and because, in general, the standards for design in China are not as high as in the Western world.

\section{Technical communicators' localization activities}

The previous results show that the interviewees considered it important to possess cultural skills to be able to function in today's globalized field of technical communication. They also stated that localization of user documentation is important. Overall, the majority of them perceived several differences between Chinese and Western manuals and they assumed that Chinese and Western users would have different expectations when using manuals. However, when asked about their localization activities, they acknowledged that they do not thoroughly localize manuals. Localization is mostly restricted to relatively small and superficial adaptations, such as small adaptations to visuals to ensure that they are not offensive for users and to textual adaptations on sentence level, to ensure that the word order corresponds with reader expectations:

We don't change the sequence of paragraphs. Usually, we change the order of sentences or we break a sentence into several ones, depending on the local users’ reading habits. (\#02, Female, TW, 6 years of experience)

Several explanations were given for the absence of more extensive localization activities. Two interviewees were of the opinion that localization of manuals is not strictly necessary because a 'good' manual can be used by users from all different cultures. However, most interviewees thought that localization of manuals would be 
useful, but they experienced several obstacles that prevented them from localizing manuals thoroughly.

Eleven interviewees referred to style sheets that they were obliged to use, so that all manuals of the international organizations they worked for were similar. Some of them referred to machine translation software that was used in their organization as well, which restricted their possibilities to localize:

Manuals are translated by the machine translation software. When editing, sentences are separated into segments by tools, and localizers or writers have little opportunities to change things. (\#11, Female, 7 years of experience)

Four interviewees explained that they did not have many possibilities to localize, because of limited budgets:

Accuracy is always prioritized. Adaptations to the local culture does not affect accuracy. So, few companies take cultural differences into account in their work. (\#06, Male, TW, 10 years of experience)

In addition to this, they said that many Chinese organizations do not regard manuals to be very important since they assumed that Chinese users did not rely on manuals. So, these organizations did not consider cultural differences and localization at all.

Apart from organizational decisions and practices that prevented the interviewees from thoroughly localizing manuals, seven interviewees acknowledged that they did not feel confident about how to localize manuals because of a lack of knowledge. They expressed their need for more knowledge about preferences of different cultural groups of users.

It's just my personal feeling. However, I find it fundamental to know why Chinese and Western users have different preferences or would perform differently. This would be better than depending on studies that only focused on Western users. (\#17, Female, 5 years of experience)

\section{CONCLUSIONS}

The interviews gave good insights in Chinese technical communicators' perceptions about cultural differences between manuals and in how these perceived differences influenced their design and localization practices. The majority of the interviewees considered cultural skills to be important for today's technical communicators who work in international environments. They noticed many differences between Chinese and Western culture that affected the field of technical communication. In their opinion, Western manual users had different expectations from manuals than Chinese users. Western users were supposed to rely on manuals to be able to operate a device effectively, while Chinese users were supposed to rely more on the assistance of other people than on the information in manuals. In line with this, the interviewees told us that they had noticed that Western manuals were longer and more detailed, and that Chinese manuals had an entertaining function as well as an informative function.

Based on these results, it would be expected that Chinese technical communicators would localize many aspects of Western manuals. But, surprisingly, the interviewees' localization activities were limited to adapting several superficial aspects. Although the selection of interviewees may not have been representative for all technical communicators in China, this is a remarkable result that deserves to be studied more intensively.

The selection of interviewees may not have been representative because almost all of them worked in software and telecommunication companies. In these internationally oriented companies, technical communicators had to use standardized tools and style sheets, so they had limited opportunities to localize. It may be that this is not the case in other sectors or types of companies, as was assumed by the interviewees themselves.

Another reason mentioned by the interviewees for not thoroughly localizing was that Chinese companies in general did not seem to consider user documentation as an important addition to a device or software program, so they did not allocate money or time to be spend on localizing. Furthermore, technical communicators experienced a lack of knowledge about how to localize and about the users' expectations and ability to use manuals. So, even if they had time to localize, they were not always able to do this.

Based on the interview results, it may be concluded that Chinese manuals will become more and more similar to Western manuals, because, firstly, Chinese companies do not consider it important to spend money on localizing; secondly Western tools and style sheets are used in Chinese organizations; and thirdly because most research about users' needs and wishes regarding user manuals is based on the Western culture. Since the majority of the technical communicators that were interviewed thought that it would be beneficial for users if manuals were adapted to their culture, more research needs to be done to provide them with knowledge on how to effectively localize. Although there is some academic literature about how to localize user documentation for Chinese users, most of these studies are small-scale studies that were published more than a decade ago. So, more research, using actual insights about cultural differences and users' expectations and abilities is needed.

However, it is hard to predict to what extent it will be problematic if manuals cannot be fully localized. Although most interviewees considered localization to be important and some even thought that improper localization could make it impossible for companies to enter international markets, some interviewees assumed that 'good' manuals 
can be used effectively by users from different cultures. Arguments for both opinions can be found in the literature. Overall, localization of manuals is assumed to be important, but there is also some evidence that Chinese and Western users are able to use manuals from both cultures equally well [13]. Hopefully, future research will shed light on the effects of localization user documentation.

\section{REFERENCES}

[1] C. M. Barnum and H. Li, "Chinese and American technical communication: A cross-cultural comparison of differences," Tech. Commun., vol. 53, no. 2, pp. 143-166, 2006.

[2] J. M. Ulijn and J. B. Strother, "The influence of culture on information overload," in Information Overload: An International Challenge For Professional Engineers And Technical Communicators, J. B. Strother, J. M. Ulijn, Eds. John Wiley \& Sons, 2012, pp. 79-101.

[3] K. St.Amant, "Introduction to the special issue - Cultural considerations for communication design: Integrating ideas of culture, communication, and context into user experience design,” Commun. Des. Q. Rev., vol. 4, no. 1, pp. 6-22, 2016.

[4] K. St.Amant, "Considering China: A perspective for technical communicators,” Tech. Commun., vol. 48, no. 4, pp. 385-388, 2001.

[5] Y. Wang, Y. Jiang, and D. Wang, “Culture and text-graphic relationship - A study of automotive service literature from China and Germany,” in Proc. IEEE Int. Professional Commun. Conf., 2007, pp. 1-8.

[6] H. Sun, "Why cultural contexts are missing: A rhetorical critique of localization practices," in Proc. 49th Annual Conf. of the Society for Tech. Commun., 2002.

[7] Y. Wang and D. Wang, "Cultural contexts in technical communication: A study of Chinese and German automobile literature,” Tech. Commun., vol. 56, no. 1, pp. 39-50, 2009.

[8] Q. Dong, “Cross-cultural considerations in instructional documentation: contrasting Chinese and U.S. home heater manuals,” ACM 25th Int. Conf. Des. Commun., pp. 221-228, 2007.

[9] Q. Wang, “A cross-cultural comparison of the use of graphics in scientific and technical communication," Tech. Commun., vol. 47, no. 4, pp. 553-560, 2000.

[10] Q. Li et al. "Cultural Differences between Chinese and Western User Instructions: A Content Analysis of Manuals for Household Appliances,” under review.

[11] D. D. Ding, "The emergence of technical communication in China Yi Jing - (I Ching) the budding of a tradition,” J. Bus. Tech. Commun., vol. 17, no. 3, pp. 319-345, 2003.
[12] Q. Li et al. "Entertaining or functional: An analysis of visuals in Chinese and Western household appliance manuals," in Proc. ProComm, IEEE Int. Professional Commun. Conf., 2018.

[13] Q. Li et al. "Cultural Differences and User Instructions: Effects of a Culturally Adapted Manual Structure on Western and Chinese Users," in Techn. Commun., vol. 62, no. 3, pp. 163-182, 2015.

\section{ABOUT THE AUTHORS}

Qian Li is a $\mathrm{PhD}$ student at the University of Twente, Enschede (The Netherlands). Her research interests concern cultural differences in technical communication.

Joyce Karreman is an assistant professor of Technical Communication at the University of Twente, the Netherlands. Her research interests include the design of instructive documents, user-centered design, and intercultural issues related to usability and user experience.

Menno D. T. de Jong is a full professor of Communication Science at the University of Twente (The Netherlands). His main research interests include technical communication (usability, user experience, and the appropriation of technology) and organizational communication. 